\title{
Transient Blue Skin: Pseudochromhidrosis
}

\author{
Johanna Eva Constance Burggraaff ${ }^{a}$, Gabor E Linthorst ${ }^{b}$, Jacobien Jeltje Hoogerwerf ${ }^{a}$ \\ ${ }^{a}$ Department of Internal Medicine, Academic Medical Center, Amsterdam, The Netherlands \\ b Department of endocrinology and metabolism, Academic Medical Center, Amsterdam, The \\ Netherlands
}

\begin{abstract}
:
Objectives: Pseudochromhidrosis is a rare condition where colours due to chromogenic microbial products or extrinsic chemicals are excreted with sweat. Chromhidrosis is the production of coloured sweat from apocrine or eccrine sweat glands. The aim of this case report is to illustrate all the steps involved in the diagnosis of pseudochromhidrosis.
\end{abstract}

Materials and methods: A 17-year-old patient with pseudochromhidrosis is presented.

Results: Clinical features of the patient were consistent with pseudochromhidrosis.

Conclusions: The distinction between chromhidrosis and pseudochromhidrosis can be made based on a detailed history, skin biopsy and empiric treatment.

Keywords: Skin, discolourization, cyanosis

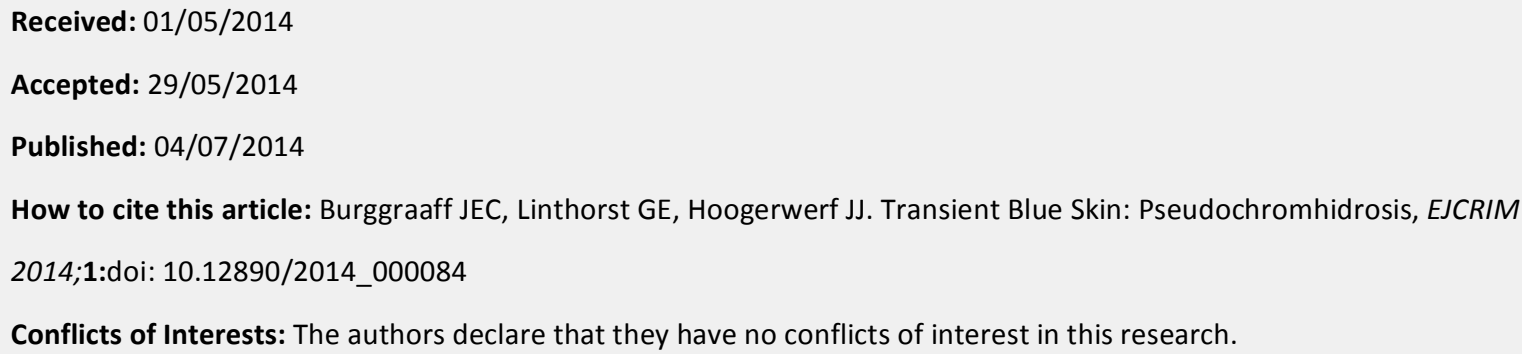




\section{Introduction}

Coloured sweat is a rare condition that can be caused by apocrine or eccrine chromhidrosis or pseudochromhidrosis. Apocrine chromhidrosis is an idiopathic disorder characterized by the increased presence of oxidized lipofuscin granules in the apocrine sweat glands and the excretion of lipofuscin in sweat ${ }^{1}$. The colour of the sweat varies depending on the level of oxidation of lipofuscin, correlating with dark staining ${ }^{2}$. In eccrine chromhidrosis, ingested water-soluble pigments are excreted in sweat ${ }^{3}$. Pseudochromhidrosis is a condition where normal colourless sweat is excreted, which becomes coloured after excretion onto the skin surface following contact with chromogenic microbial products or extrinsic chemicals ${ }^{4}$. Malassezia furfur and Bacillus spp. have been considered as causes of blue pseudochromhidrosis ${ }^{5}$. In this case report, we describe a 17-year-old girl referred to our hospital because of recurring blue skin discoloration, which disappeared after treatment with an antiseptic scrub.

\section{Case report}

A 17-year-old girl was referred to our hospital because of unexplained recurring transient blue skin discoloration of the neck and hands, and occasionally of her chest, back and limbs. This was unrelated to cold or exertion, but improved after showering and would disappear after several hours to days. In addition, she mentioned chronic fatigue, slight dyspnoea on exertion and lightheadedness, as well as aching of the head, joints and stomach. She had dropped out of school because of these complaints. Her medical history included empiric treatment with vitamin B12 at her own request. The patient's mother and grandmother were affected by fibromyalgia.

Physical examination showed non-blanchable blue macules (Fig. 1), which could be scraped off (Fig. 2) and were not present under her armpits and undergarments. The colour of tears, saliva and urine was unchanged.

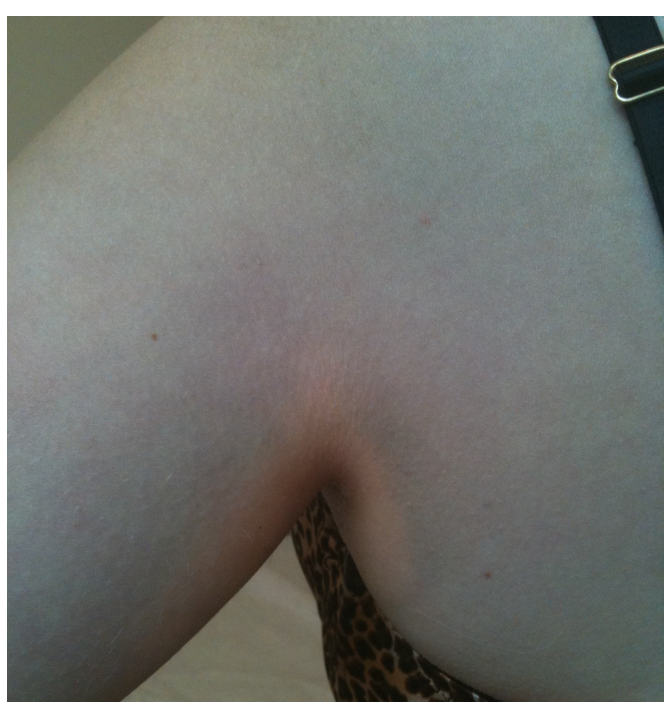

Figure 1: Non-blanchable blue macules of the neck and shoulders

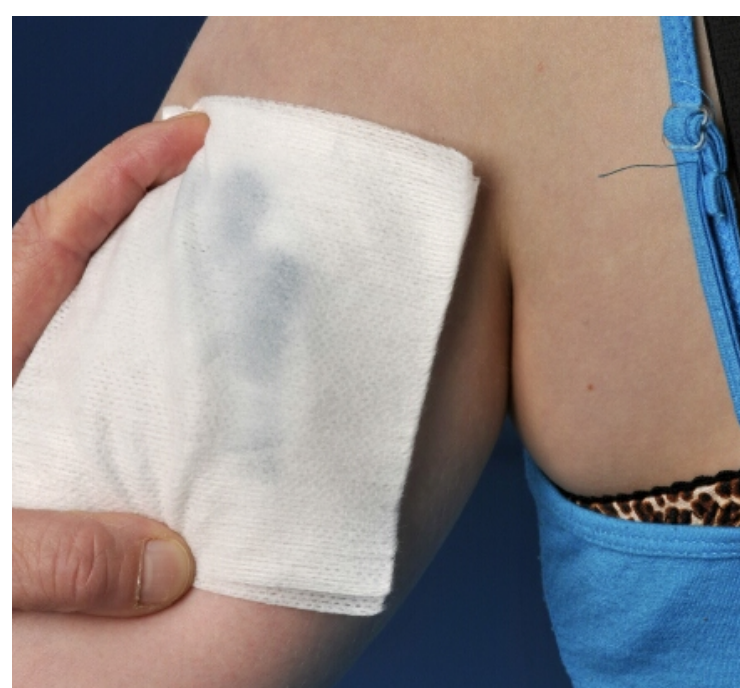

Figure 2: Skin scrapings obtained from our patient. 
Extensive laboratory tests and examinations to identify a cause of presumed cyanosis were all normal (erythrocyte sedimentation rate [ESR], full blood count, coagulation, methaemoglobin, cardiac ultrasound, antibodies for several autoimmune disorders and capillary microscopy). More detailed questioning ruled out ingestion of pigments, and a skin biopsy was negative for lipofuscin pigment granules, which excluded chromhidrosis.

Empiric treatment with chlorhexidine scrub resulted in the complete disappearance of the blue discoloration, which was suggestive of pseudochromhidrosis. Her other complaints persisted.

\section{Discussion}

Although (pseudo)chromhidrosis does not constitute a health issue, it may cause psychological stress and social embarrassment, as demonstrated by this case report. In addition, patients can also undergo unnecessary diagnostic procedures before (pseudo)chromhidrosis is considered.

Apocrine chromhidrosis is diagnosed by the presence of lipofuscin granules in a skin biopsy and autofluorescence of skin specimens or clothing, while diagnosis of eccrine chromhidrosis requires the exclusion of ingestion of pigments with a detailed patient history. Pseudochromhidrosis is a clinical diagnosis based on exclusion of chromhidrosis and successful treatment with either antibiotics or, as in this case, an antiseptic scrub4.

The distinction between apocrine or eccrine chromhidrosis and pseudochromhidrosis is important for the prognosis. Apocrine chromhidrosis is usually recurrent because there is no known treatment that targets the accumulation of lipofuscin ${ }^{1}$. Therefore, the treatment is usually targeted towards reducing sweat secretion, either by topical capsaicin cream, $20 \%$ aluminium chloride hexahydrate solution (Drysol) or injections of botulinum toxin ${ }^{1}$. Although there is no long-term follow-up of patients with chromhidrosis, it is likely that the symptoms may diminish with age due to natural regression of the apocrine sweat glands ${ }^{1}$.

Treatment of eccrine chromhidrosis consists only in ceasing the consumption of soluble pigment. Pseudochromhidrosis can be treated with antibiotics, either topical or systemic. This usually results in complete clearance of the chromogenic agent, although in some cases it persists ${ }^{4}$. As shown in this case report, topical antiseptics can also be effective.

\section{Learning Points}

- Physical examination of patients with coloured skin should include determining whether the colour can be scraped off.

- Pseudochromhidrosis can be treated with antiseptic scrub instead of topical or systemic antibiotics. 


\section{References}

1. Wang A, Wysong A, Nord KM, Egbert BM, Kosek J. Chromhidrosis: a rare diagnosis requiring clinicopathologic correlation, Am J Dermatopathol 2013 Epub Mar 14.

2. Thami GP, Kanwar AJ. Red facial pseudochromhidrosis, Br J Dermatol 2000;142:1219-1220.

3. Cilliers J, de Beer C. The case of the red lingerie: chromhidrosis revisited, Dermatology 1999;199:149-152.

4. Harada K, Morohoshi T, Ikeda T, Shimada S. A patient with pseudochromhidrosis presenting with pink nails, J Am Acad Dermatol 2012;67:e74-75.

5. Castela E, Thomas P, Bronsard V, Lacour JP, Ortonne JP, Passeron T. Blue pseudochromhidrosis secondary to topiramate treatment, Acta Derm Venereol 2009;89:538-539. 